\title{
Topological Identification Criteria, Stability, and Relevance of Pentagonal Nanochannels in Amorphous Ice
}

\author{
Nandlal Pingua and Pankaj A. Apte* \\ Department of Chemical Engineering, Indian Institute of Technology Kanpur, Kanpur \\ Uttar Pradesh India-208016 \\ E-mail: papte@iitk.ac.in
}

Phone: +91 512 2597629. Fax: +91 5122597629

SUPPLEMENTARY INFORMATION 


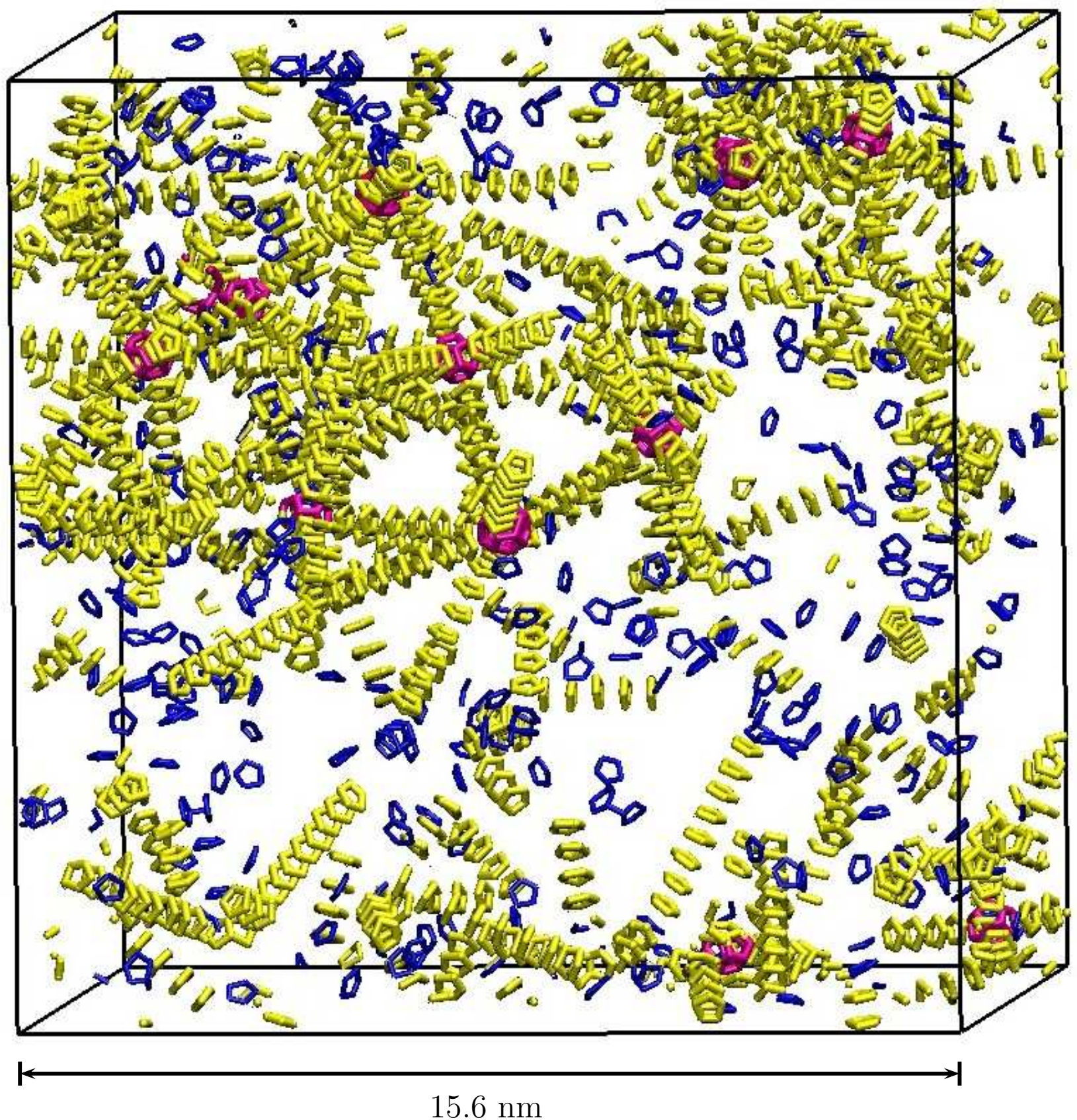

Figure S1. PNCs, dodecahedron cages and additonal flat pentagonal rings in amorphous ice: The snapshot in terms of S-,D1-,D2-,D3- PNCs (all are in yellow color), the flat pentagonal rings (which are not the part of channels) in blue color and dodecahedron cages (in pink color) in amorphous ice at X1 location for isothermal non-optimal-1 trajectory (see Figure. S4). The flat rings are chosen based on the criteria mentioned in the main text. 


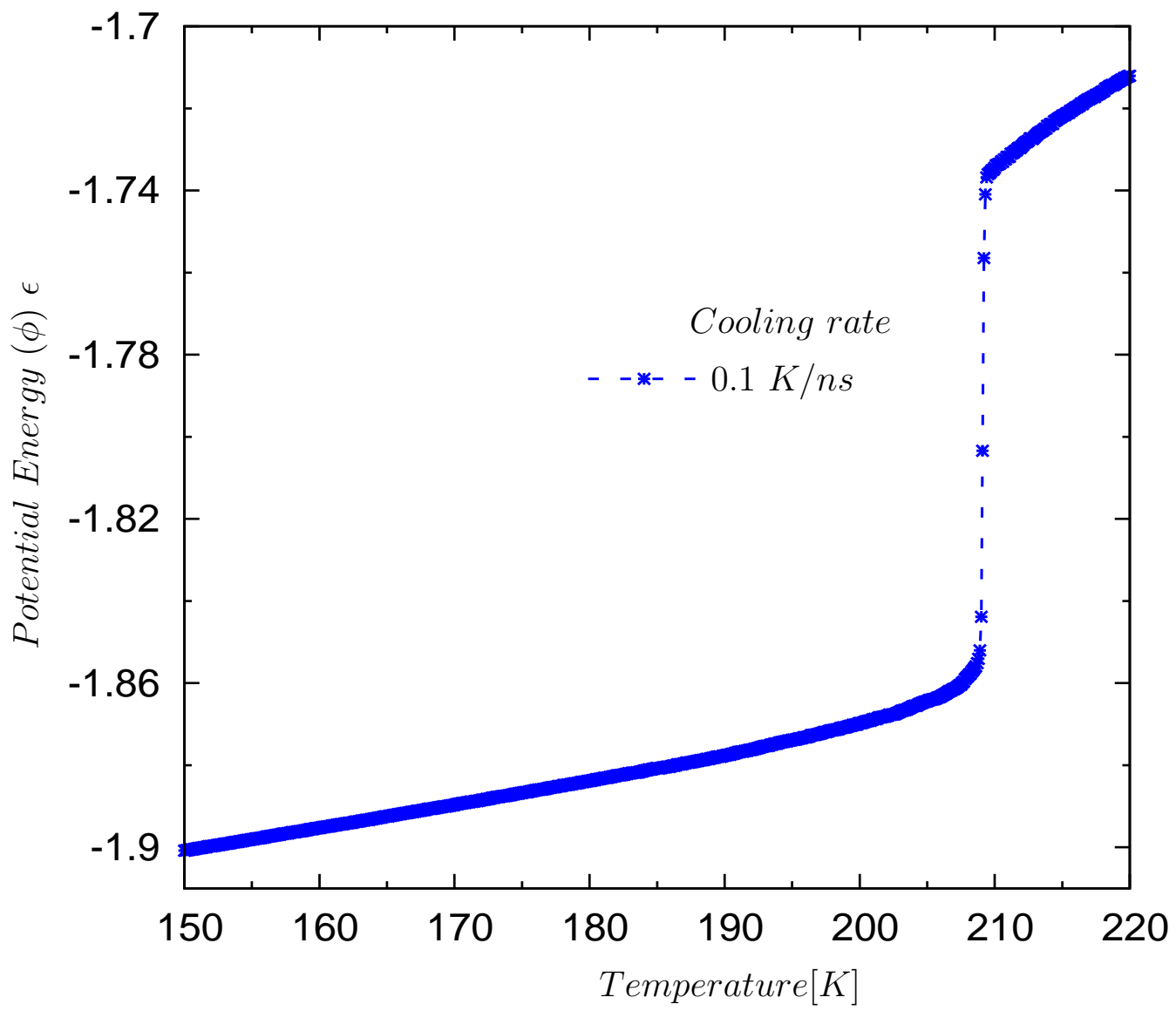

Figure S2. Evolution of per particle potential energy $(\phi)$ from 220 to $150 \mathrm{~K}$ at constant cooling rate $0.1 \mathrm{~K} / \mathrm{ns}$. 

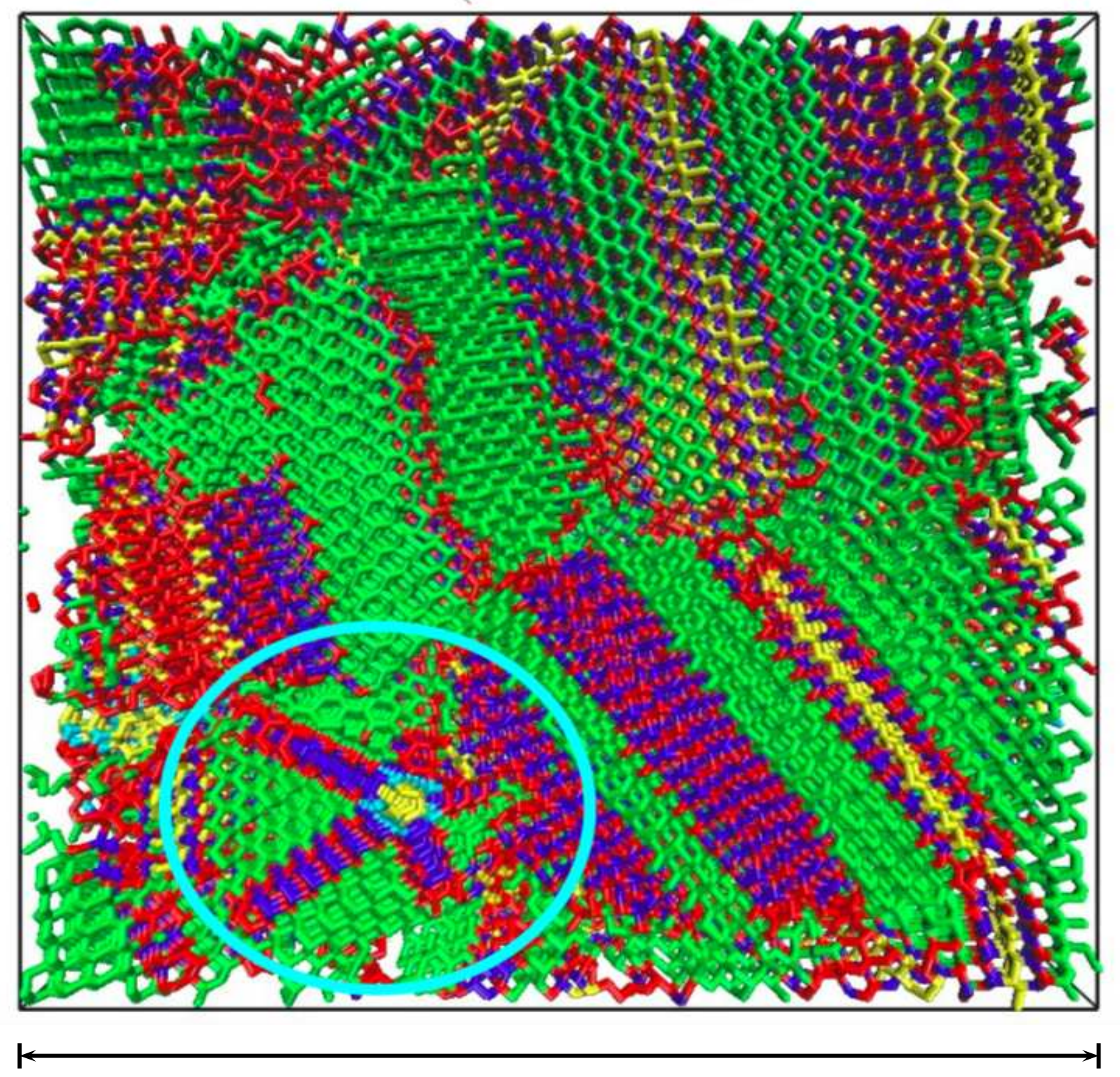

$16.2 \mathrm{~nm}$

Figure S3. Cubic-Hexagonal stacking layers in amorphous ice: The Snapshot of cubic-hexagonal stacking obtained in the amorphous state at $150 \mathrm{~K}$ during isobaric cooling with a rate $0.1 \mathrm{~K} / \mathrm{ns}$ (see Figure S2). The same color sheme as in Fig. 4 of the main text is used. The circled region show the PNC with an 'extended' five-fold symmetric environment (see Figure.4(a)). 


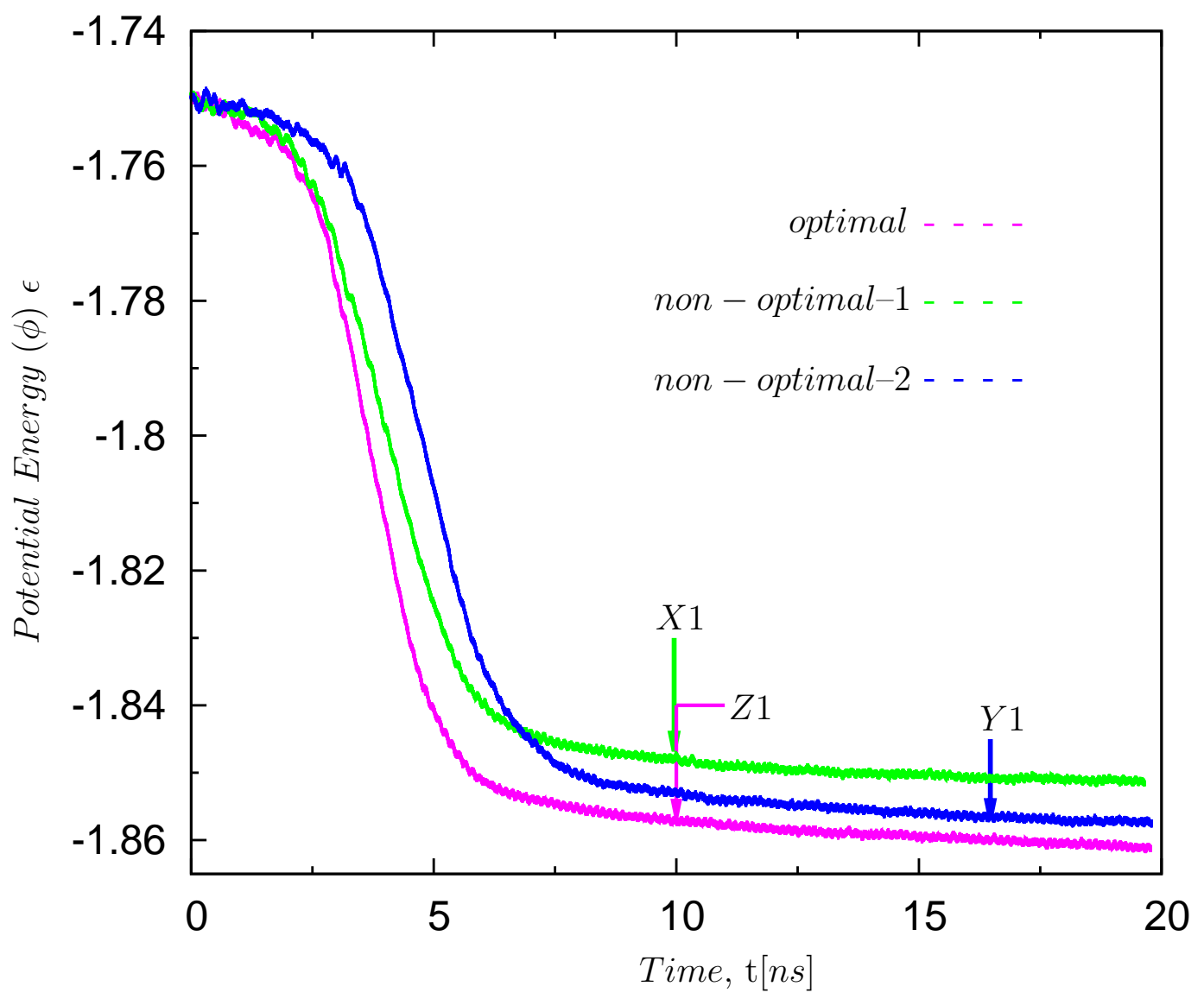

Figure S4. The per particle potential energy $(\phi)$ obtained during the isothermal-isobarice (optimal and non-optimal) trajectories of $\mathrm{mW}$-water at $\mathrm{T}=205 \mathrm{~K}$. The locations $X 1, Y 1$, and $Z 1$ along the trajectories are marked for references in the main text and other figures. 


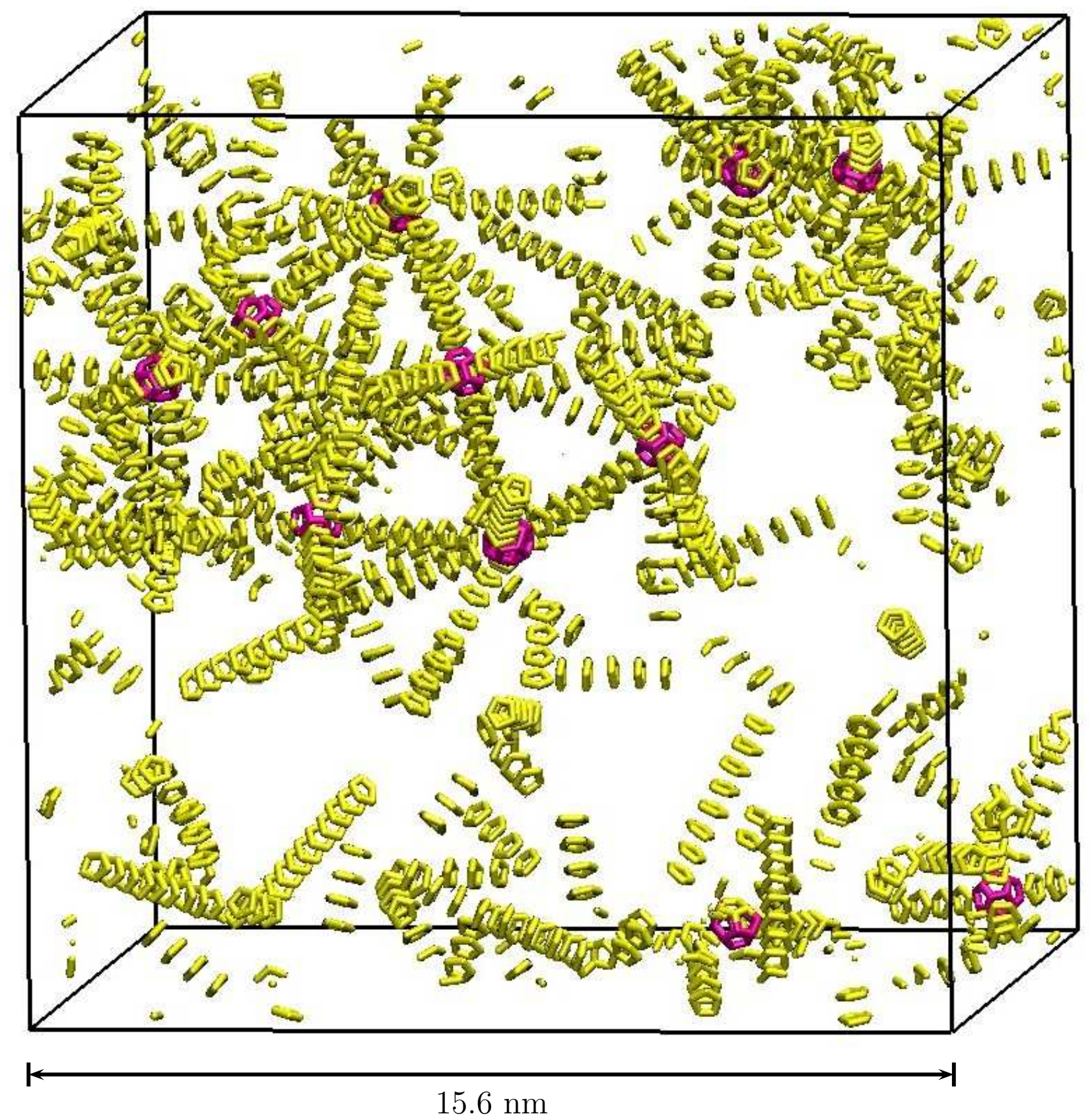

Figure S5. PNCs and dodecahedron cages in amorphous ice: The snapshot in terms of S-,D1-,D2-,D3-PNCs (all are in yellow color) and dodecahedron cages (pink color) taken at X1 location of isothermal non-optimal-1 trajectory (see Fig. S4). A total of 11-dodecahdron cages are observed. 


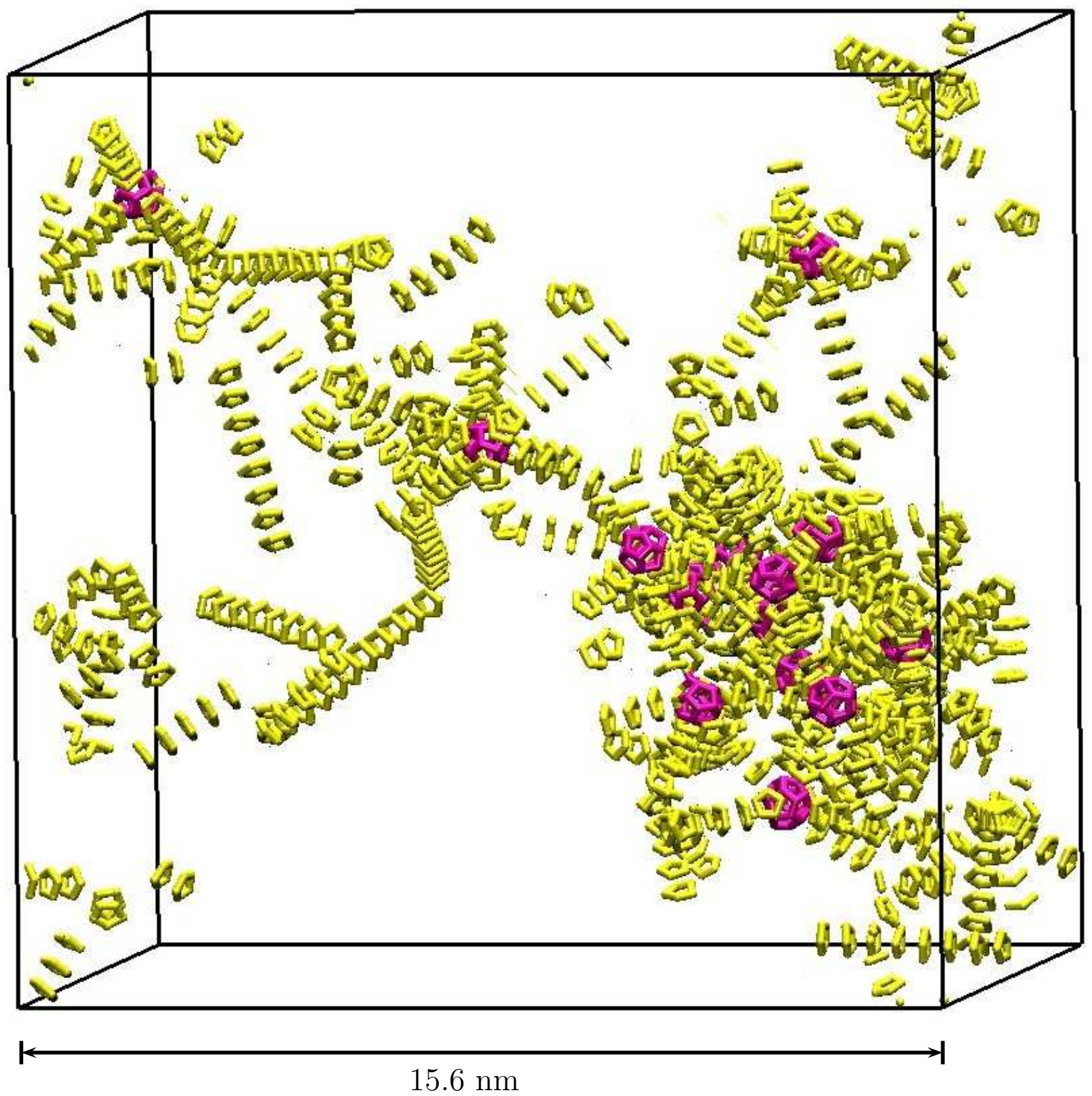

Figure S6. PNCs and dodecahedron cages in amorphous ice: The snapshot in terms of S-,D1-,D2-,D3-PNCs (all are in yellow color) and dodecahedron cages (pink color) taken at Y1 location of isothermal non-optimal-2 trajectory (see Fig. S4). A total of 15-dodecahedron cages are observed. 


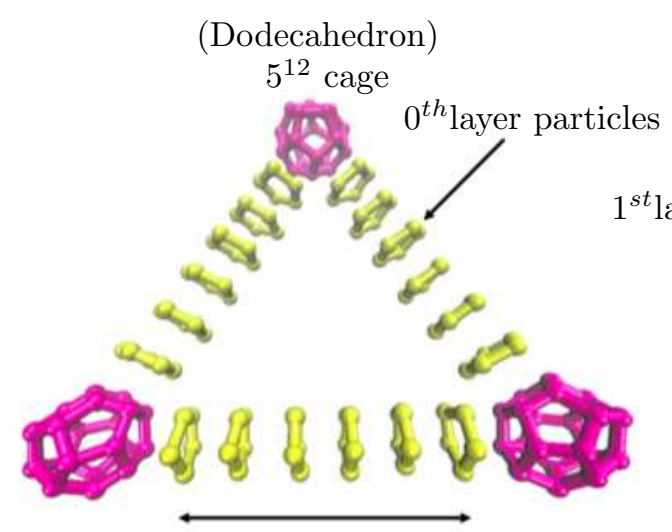

Symmetric

pentagonal nanochannel

(a)

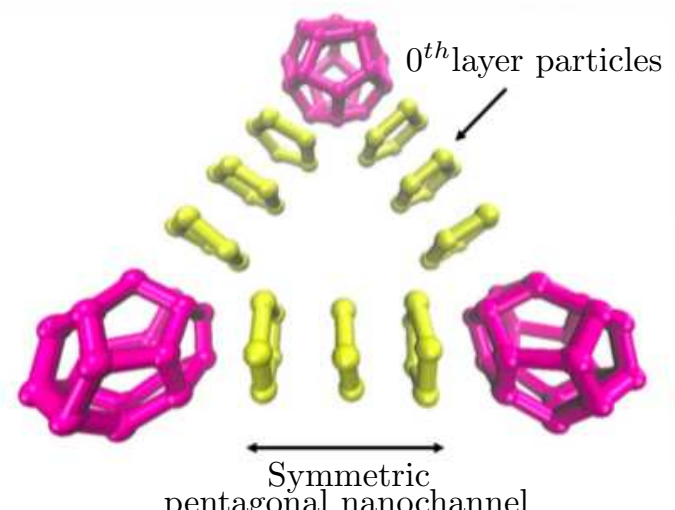

pentagonal nanochannel

(d)

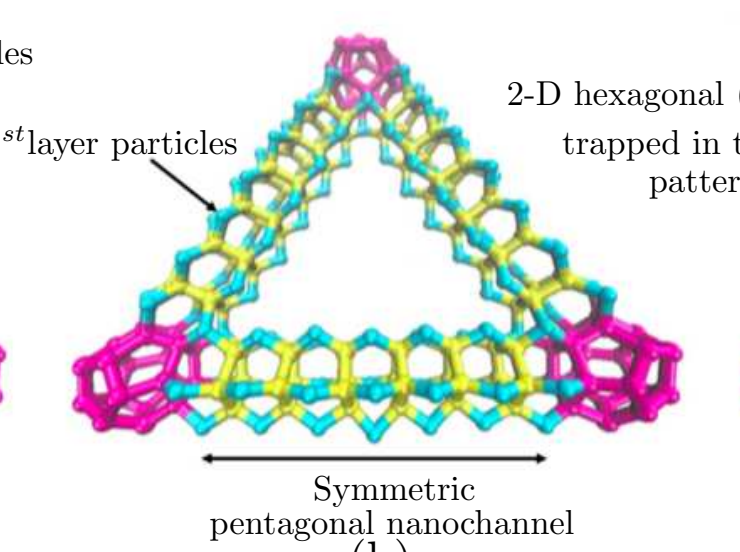

(b)

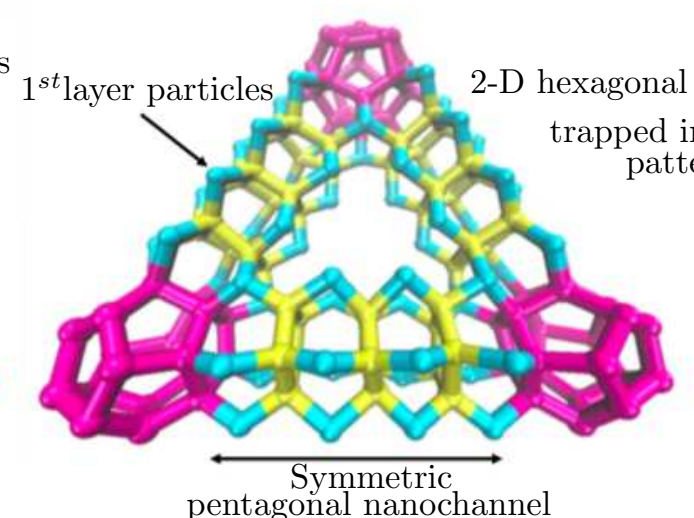

(e)

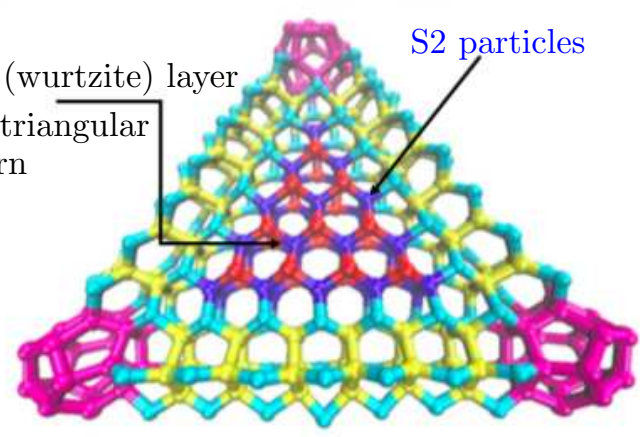

(c)

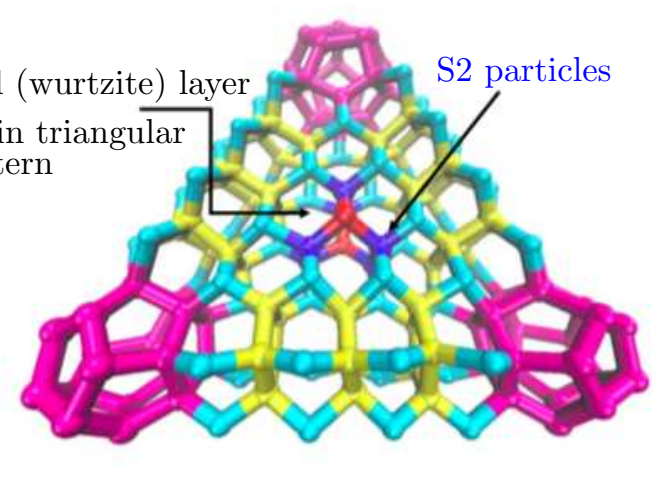

(f)

Figure S7. Triangular pattern and trapped 2-D hexagonal crystalline monolayers: The upper pannel (a)-(c) depict the larger triangular patterns (each channel consists of 6pentagonal rings), the lower pannel (d)-(f) depict the smaller triangular patterns (each channel consists of 3-pentagonal rings) and trapped 2-D hexagonal (wurtzite) monolayer in triangular pattern. 


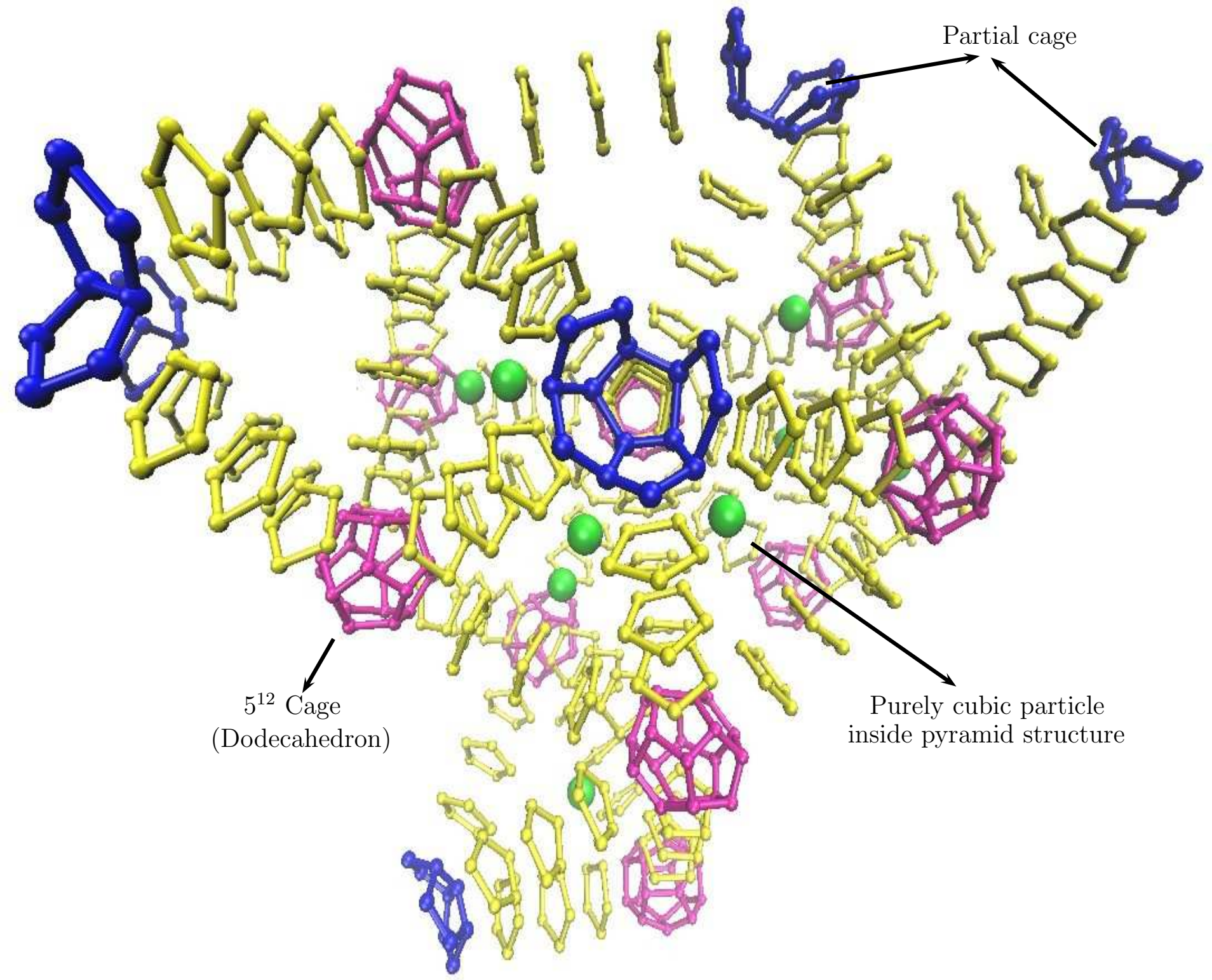

Figure S8. A network of pyramids formed by PNCs and the dodecahedron cages: This is the same network as in Fig. 7(b) of the main text, but with a different orientation. There are several partial cages (in blue color), which also serve as vertices of the pyramid patterns in the network. 


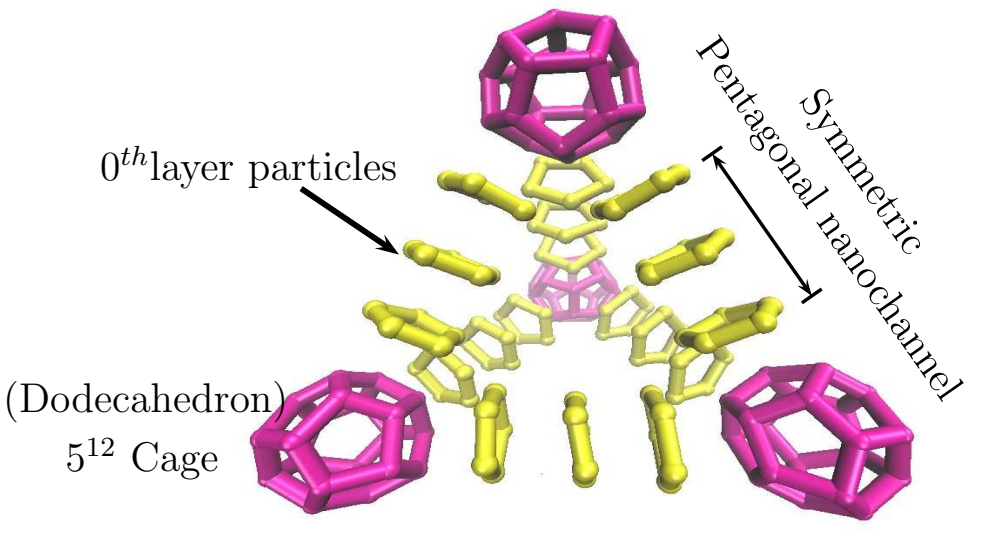

(a)

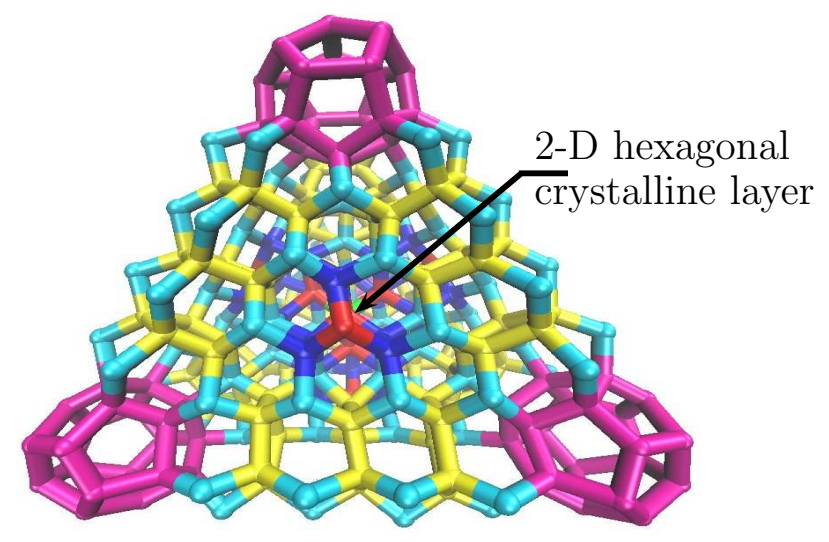

(c)

(e)

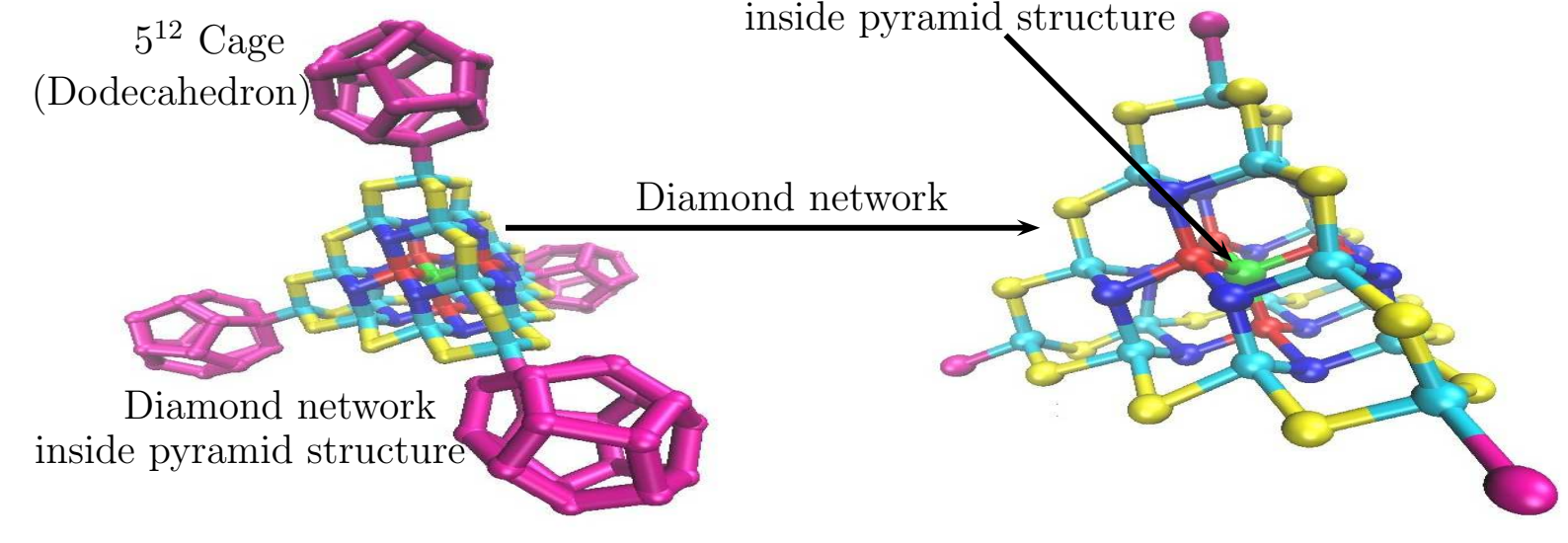

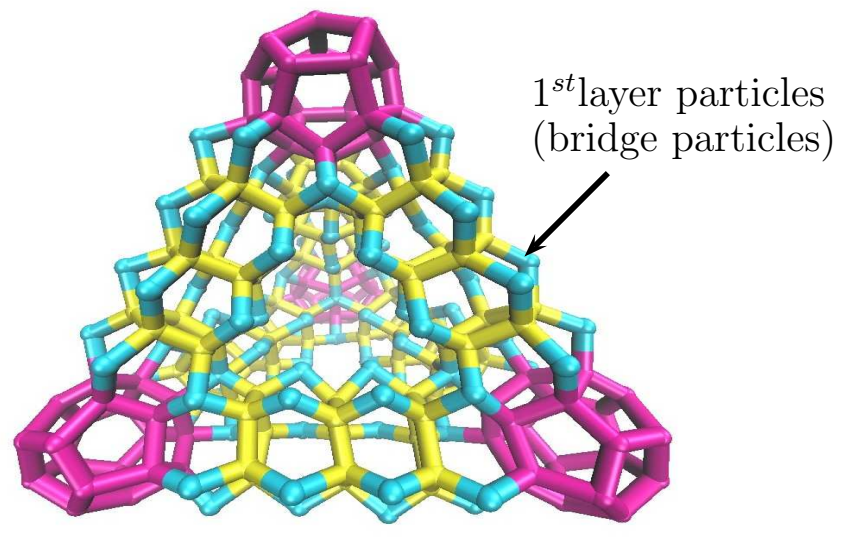

(b)

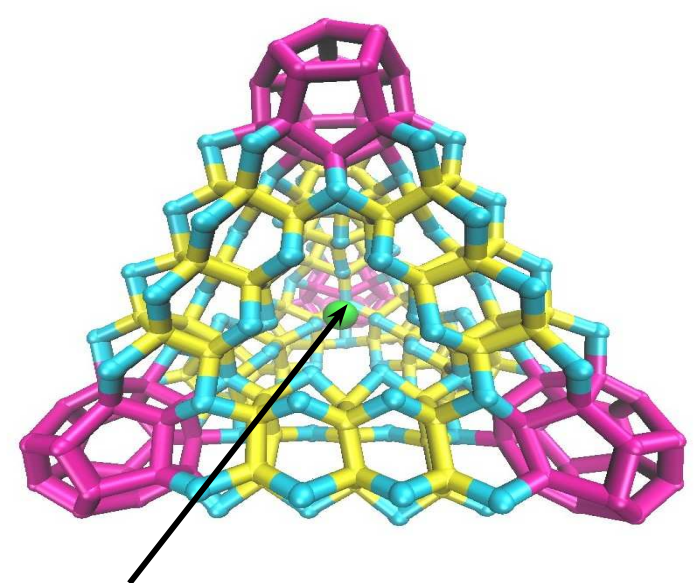

(d)

Purely cubic particle inside pyramid structure

(f)

Figure S9. Pyramid structure pattern and diamond network inside pyramid: (a) pyramid formed by PNCs and dodecahedron cages which serve as vertices of the pyramid; (b) pyramid and pentagonal rings connected with bridge particles; (c) pyramid structure inside filled with diamond network and 2-D hexgonal crystalline layers on the surface of the pyramid pattern; (d) pyramid structure with inside purely cubic central particle shown in green color; (e) diamond network with dodecahedron cages; (f) diamond network with 10-diamond unit present inside pyramid with a purely cubic central particle shown in green color. 


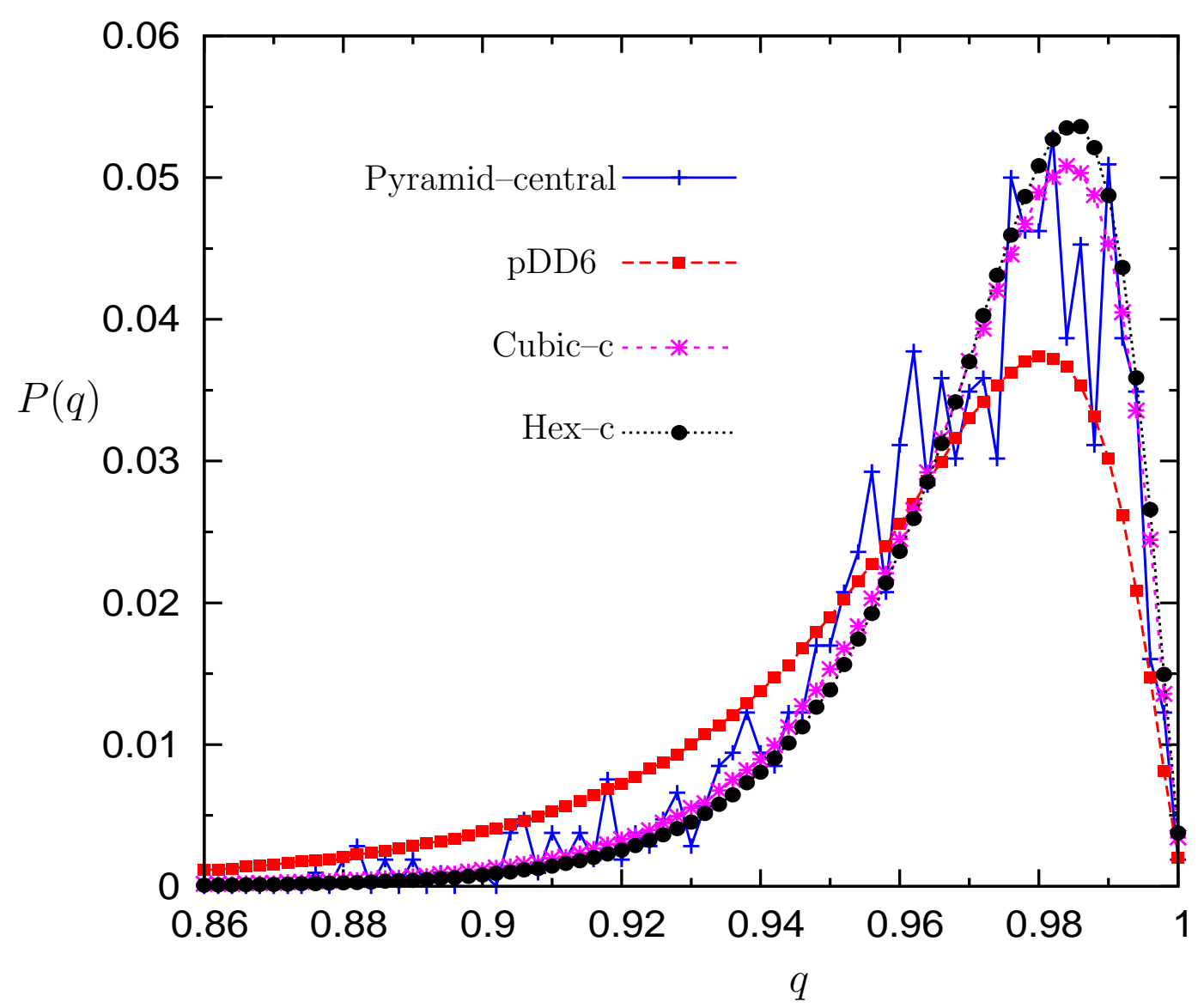

Figure S10. The tetrahedral order parameter ' $q$ ' distribution at Y1 location of isothermal non-optimal-2 trajectory (see Figure S4). The distribution of central purely cubic particles is shown together with cubic crystal (cubic-c) and hexagonal crystal (Hex-c) distribution at $\mathrm{T}=205 \mathrm{~K}$. Also shown is the distribution of all purely cubic particles (pDD6) in the amorphous state. The distributions are sampled after every $0.1 \mathrm{~ns}$ over a total time period of $15.5 \mathrm{~ns}$. Each point in these plots represents average fraction of the given class of particles with the $q$ value in the interval $(q-\Delta q, q)$, where $\Delta q=0.002$. 


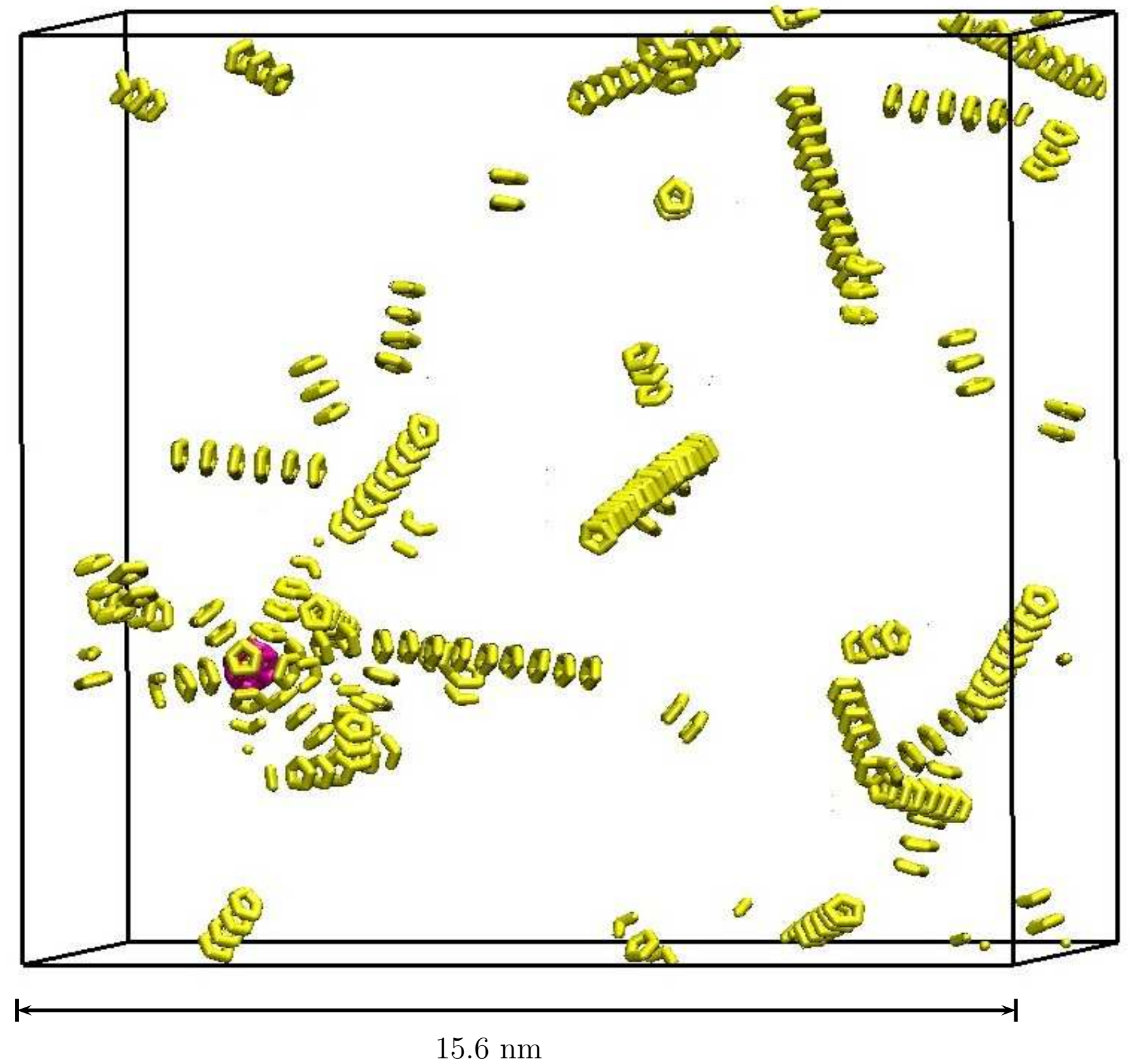

Figure S11. PNCs and dodecahedron cages in amorphous ice: The snapshot in terms of S-,D1-,D2-,D3-PNCs (all are in yellow color) and dodecahedron cages (pink color) taken at Z1 location of isothermal optimal trajectory (see Fig. S4). 


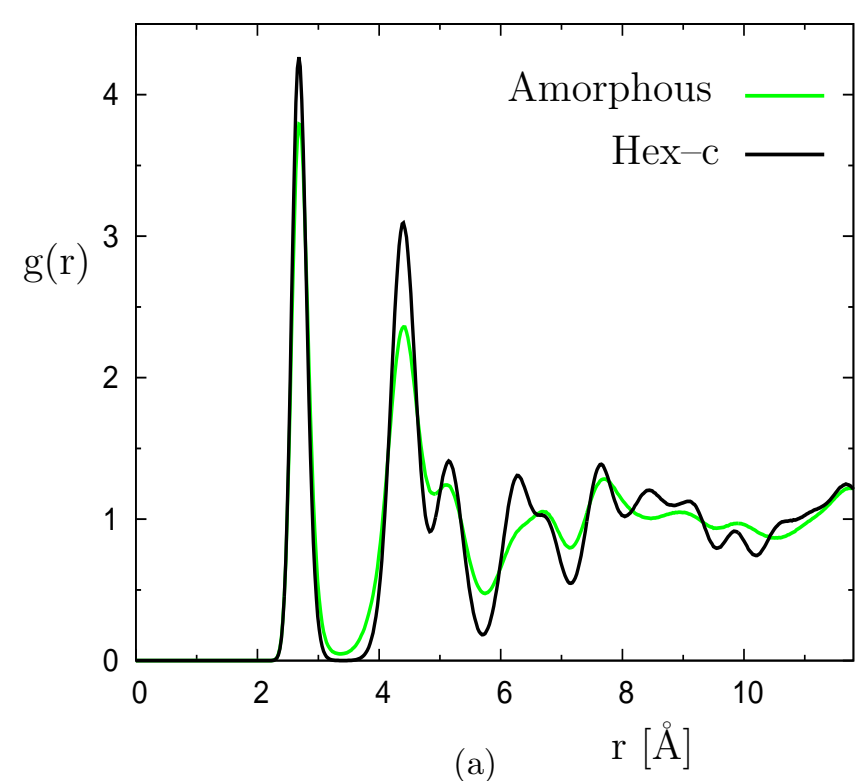

(a)

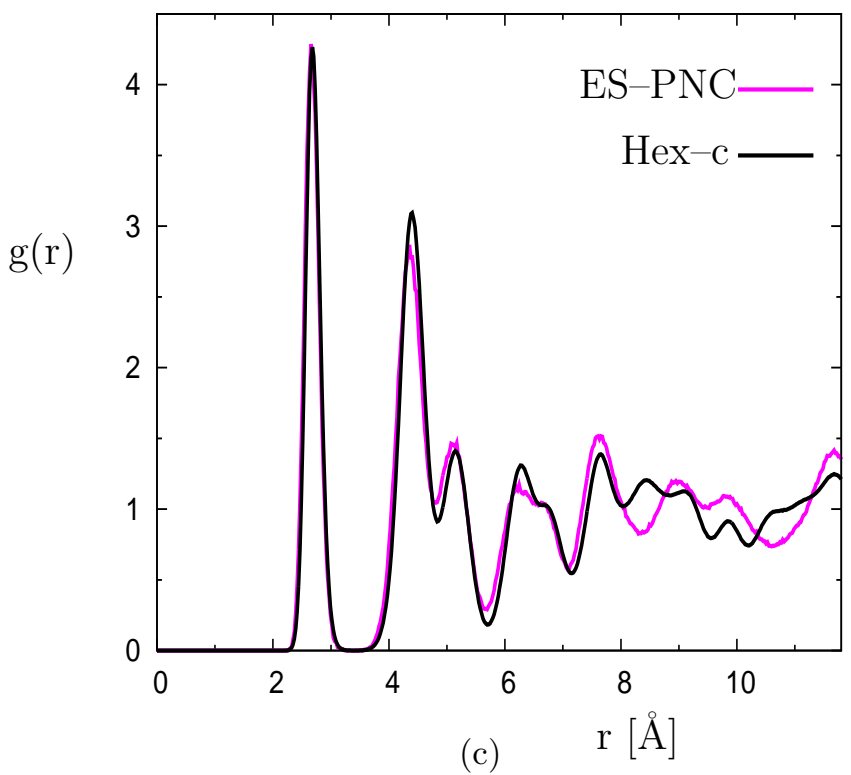

Figure S12. The radial distribution function (RDF) at $205 \mathrm{~K}$ (a) the RDF of amorphous state, (b) the partial RDF of the pentagonal ring particles of the dodecahedron $\left(5^{12}\right)$ cages, and (c) the partial RDF of pentagonal ring particles of ES-PNCs. All the distributions are obtained in non-optimal-1 trajectory at X1 location (see Fig. S4); Each of the RDFs in (a), (b), and (c) is compared with the RDF of the hexagonal crystalline phase of $\mathrm{mW}$ water at $205 \mathrm{~K}$. (d) The pictorial diagram shows a perfect tetrhedral arragement in which first, second, and third neighbor distances are 2.68, 4.38, and $6.74 \mathrm{~A}^{0}$, respectively. These closely agree with the peak positions in the RDF of amorphous state seen in (a). 